\section{Violência sexual: estudo descritivo sobre as vítimas e $o$ atendimento em um serviço universitário de referência no Estado de São Paulo, Brasil}

\author{
Sexual violence: a descriptive study of rape victims \\ and care in a university referral center in São Paulo \\ State, Brazil
}

\author{
Violencia sexual: estudio descriptivo sobre las \\ víctimas y la atención en un servicio universitario \\ de referencia en el estado de São Paulo, Brasil
}

\footnotetext{
${ }_{1}$ Faculdade de Ciências Médicas, Universidade Estadual de Campinas, Campinas, Brasil.

2 Hospital da Mulher Prof. Dr. José Aristodemo Pinotti, Universidade Estadual de Campinas, Campinas, Brasil.

Correspondência C. O. Facuri

Serviço de Saúde Dr. Candido Ferreira, Faculdade de Ciências Médicas, Universidade

Estadual de Campinas. Rua Alexander Fleming 101 Campinas, SP 13083-881,

Brasil.

claudiafacuri@yahoo.com.br
}

\begin{abstract}
Rape is a global public health problem, and steps have been taken to encourage studies on the issue and propose interventions for its prevention and appropriate care. This study aimed to characterize the population of female rape victims and describe the characteristics of the sexual assault and the care provided at a university referral center. This was a quantitative retrospective study of care provided to female rape victims from June 2006 to December 2010. The majority of the women ( $n=687)$ were white, single, had no children, with a mean age of 23.7 years and primary to secondary schooling, employed, and practiced a religion. One-fourth of the victims reported no sexual intercourse prior to the sexual assault. Rape occurred mainly at night, on the street, perpetrated by a single stranger, with vaginal penetration, and with threatened or actual force. Most of the victims had reported the rape to someone and felt supported. Early care occurred for almost $90 \%$ of women, allowing preventive measures. From 2006 to 2010 there was an increase in the proportion of women that sought help. Better knowledge of the characteristics of this group and the event itself can help improve the structure and functioning of models to assist rape victims.
\end{abstract}

Violence against Women; Rape; Sexual Violence; Sex Offenses
Cláudia de Oliveira Facuri 1 Arlete Maria dos Santos Fernandes 2 Karina Diniz Oliveira 1 Tiago dos Santos Andrade 1 Renata Cruz Soares de Azevedo 1

\section{Resumo}

A violência sexual é problema de saúde pública global e ações têm sido implementadas para estimular estudos no tema, a fim de propor intervenções de prevenção e atendimento adequado. Este trabalho objetivou caracterizar a população de mulheres que sofreram violência sexual, e descrever as características da agressão e do atendimento dispensado em um serviço universitário de referência. Estudo quantitativo e retrospectivo com atendimentos por violência sexual de junho de 2006 a dezembro de 2010. Avaliadas 687 mulheres, a maioria branca, solteira, sem filhos, com idade média de 23,7 anos, escolaridade entre fundamental e média, empregadas, com religião e prática religiosa. Um quarto sem relação sexual anterior. Violência sexual principalmente à noite, na rua, por agressor desconhecido e único, via vaginal e com intimidação. A maioria contou para outras pessoas e se sentiu apoiada. Atendimento precoce para quase $90 \%$ das mulheres, instaurando medidas profiláticas. Ocorreu aumento da procura precoce ao longo do período. Conhecer melhor as características da população e do evento pode auxiliar a estruturação e qualificação de modelos de atendimento.

Violência contra a Mulher; Estupro; Violência Sexual; Delitos Sexuais 


\section{Introdução}

A violência sexual é um fenômeno universal, no qual não há restrição de sexo, idade, etnia ou classe social, que ocorreu no passado e ainda ocorre, em diferentes contextos ao longo da história da humanidade 1,2. Embora atinja homens e mulheres, estas são as principais vítimas, em qualquer período de suas vidas, no entanto, as mulheres jovens e adolescentes apresentam risco mais elevado de sofrer esse tipo de agressão 3,4,5.

Estupro é definido pela Organização Mundial da Saúde (OMS) como todo ato sexual ou tentativa para obter ato sexual, investidas ou comentários sexuais indesejáveis contra a sexualidade de uma pessoa usando coerção ${ }^{2}$. No Brasil, é definido juridicamente como sendo o ato de "constranger alguém, mediante violência ou grave ameaça, a ter conjunção carnal ou a praticar ou permitir que com ele se pratique outro ato libidinoso" 6.

Estima-se a prevalência global de estupro de 2 a $5 \%$ e incidência de 12 milhões de vítimas a cada ano 2 . A prevalência de estupro em mulheres, ao longo da vida, corresponde a aproximadamente $20 \% 4,7$. Dados nacionais indicam uma média diária de 21,9 mulheres procurando atendimentos em serviços de saúde por violência sexual e 14,2 mulheres/dia notificadas como vítimas de estupro (Departamento de Informática do SUS. http://dtr2004.saude.gov.br/sinanweb/tabnet/ dh?sinannet/violência/bases/testbrnet_001.def, acessado em 20/Jul/2012).

A violência sexual tem efeitos devastadores nas esferas física e mental, em curto e longo prazos 2,4. Entre as consequências físicas imediatas estão a gravidez, infecções do trato reprodutivo e doenças sexualmente transmissíveis (DST) 1,2,4. Em longo prazo, essas mulheres podem desenvolver distúrbios ginecológicos e na esfera da sexualidade 5 . Mulheres com história de violência sexual têm maior vulnerabilidade para sintomas psiquiátricos, principalmente depressão, pânico, somatização, tentativa de suicídio, abuso e dependência de substancias psicoativas 5 .

É possível dividir as vítimas em duas subpopulações: uma que envolve agressor conhecido (frequentemente intrafamiliar), em que há menor taxa de procura por auxílio, maior número de atos praticados e composta por mulheres mais jovens; e outra por mulheres agredidas por estranho, faixa etária mais velha (idade média de 22 anos), maiores taxas de agressão física, de denúncia e de procura por auxílio 8,9,10.

Dada a gravidade do problema no que tange à violação dos direitos humanos e ao impacto físico, psíquico e social, a violência sexual foi reconhecida como um problema de saúde pública global em 1993, pela Organização Pan-Americana da Saúde (OPAS) e OMS 2,11. Desde então, muitas ações foram implementadas para estimular estudos sobre a violência contra a mulher e propor intervenções relativas à prevenção da agressão, atendimento adequado, humanizado e normatizado 1,12.

Atribui-se à boa assistência prestada o aumento do número de mulheres que recorrem aos serviços imediatamente após a violência sexual, com a utilização da contracepção de emergência nas primeiras horas após o evento e a consequente diminuição do número de interrupções da gravidez por estupro verificada na última década 1 .

Em função disso, é fundamental que haja serviços que atendam a essa demanda de forma ágil, acolhedora, em bom ambiente e com capacidade de atuar nas preocupações imediatas (lesão física, DST, gravidez) e nas dificuldades psíquicas $4,5,13$.

No ano de 2010, existiam no Brasil 442 serviços de saúde para atendimento de violência sexual e 60 serviços especializados para atendimento de procedimentos relacionados à violência sexual previstos em lei 14 .

Com base no cenário descrito, considera-se que prover informações sobre as características das mulheres vítimas de violência sexual, a descrição da agressão e do atendimento recebido, podem auxiliar na discussão acerca de abordagens do problema no âmbito dos serviços de saúde.

Este estudo teve o objetivo de caracterizar a população de mulheres que sofreram violência sexual, e descrever as características da agressão e do atendimento dispensado em um serviço universitário de referência do Estado de São Paulo.

\section{Métodos}

Estudo descritivo, quantitativo e retrospectivo, que avaliou o perfil sociodemográfico, as características da agressão e do atendimento de mulheres vítimas de violência sexual avaliadas em um serviço universitário de referência, no período de junho de 2006 a dezembro de 2010.

Foram excluídas mulheres pré-púberes, com idade inferior a 12 anos (atendidas em Ambulatório de Violência Contra a Criança da mesma universidade) e mulheres contra as quais não foi configurada violência sexual após triagem. Nas mulheres vitimizadas em mais de um evento de violência sexual no mesmo período, foi considerado apenas o primeiro atendimento.

O trabalho foi realizado no Hospital da $\mathrm{Mu}-$ lher Prof. Dr. José Aristodemo Pinotti da Univer- 
sidade Estadual de Campinas (Unicamp), serviço terciário universitário especializado na assistência à saúde da mulher e do recém-nascido, e referência do município e da região metropolitana de Campinas que abrange mais de 17 cidades, com cobertura estimada em torno de 2,5 milhões de pessoas. A assistência a mulheres vítimas de violência sexual foi instituída a partir de 1994, oferece atendimento de emergência e ambulatorial multidisciplinar (ginecologista, enfermeiro, psicólogo, assistente social e psiquiatra) visando a prevenir a gravidez, as DST e promover a recuperação física, psicológica e social da mulher, e inclui a assistência à gestação decorrente de estupro em acordo com as normas preconizadas pelo Ministério da Saúde 15,16.

O primeiro contato com o serviço é realizado por enfermeiros, que fazem os encaminhamentos de acordo com o tipo de violência. As mulheres são avaliadas por enfermeiro e médico 17, e as ocorrências são classificadas em dois tipos segundo o intervalo de tempo transcorrido entre a agressão e o contato com o serviço de saúde: imediatas (até cinco dias após a violência sexual) e tardias (após cinco dias da agressão) 17. Depois desse atendimento de emergência, as pacientes são encaminhadas para atendimento ambulatorial com equipe multiprofissional e seguidas por seis meses.

Os dados foram coletados com base nas informações dos prontuários dos atendimentos multidisciplinares, em ficha de coleta desenhada para o estudo. Foi realizada uma fase piloto por meio do levantamento de 50 fichas visando à adequação do registro dos dados.

Foram levantadas as seguintes variáveis: - Sociodemográficas: idade, cor da pele, estado civil, prole, escolaridade, situação profissional (sendo que a categoria "empregada" foi utilizada quando a paciente estava em atividade laboral regular que não a atividade de dona de casa), religião, prática religiosa, ter tido relação sexual anteriormente ao evento, se tinha antecedente pessoal ou familiar de violência sexual e se possuía antecedente de doença crônica;

- Relacionadas à violência sexual: horário do evento, local de abordagem, características do agressor (conhecido ou desconhecido, único ou múltiplos), presença e tipo de intimidação (arma de fogo, arma branca, força física, ameaça verbal), tipo de agressão sexual (coito vaginal, anal, oral ou mais de um tipo) e realização de Boletim de Ocorrência (BO); e

- Relacionadas ao atendimento de emergência: tempo transcorrido entre o evento e a primeira avaliação no serviço (calculado baseando-se nas informações apresentadas na ficha de atendimento em até 24 horas ou mais de 24 horas), classificação do atendimento (imediato: até cinco dias, ou tardio: após cinco dias), prescrição de anticoncepção de emergência, profilaxia com antirretrovirais, vacina ou imunoglobulina contra hepatite B, profilaxia para DST bacterianas, se contou a alguém e quem (cônjuge (namorado ou marido), mãe, pai, irmãos, amigos, polícia, outros), se sentiu-se apoiada (sim, não ou parcialmente).

Para avaliação do seguimento foram definidas duas variáveis: não adesão (pacientes que compareceram apenas ao atendimento de emergência) e adesão (mulheres que compareceram ao atendimento ambulatorial).

As taxas para tempo transcorrido entre a agressão e o primeiro contato com o serviço de referência (24 e 72 horas) e realização de BO foram apresentadas em agrupamentos de 30 meses (2006-2008) e 24 meses (2009-2010) para melhor comparação do comportamento dessa população ao longo do período.

Foram incluídos todos os prontuários relativos aos atendimentos do período acima definido. Os dados coletados foram inseridos em banco de dados usando-se o programa SPSS 11.5 (SPSS Inc., Chicago, Estados Unidos) e submetidos à revisão e análise estatística. A comparação entre os períodos de tempo foi realizada com o teste de qui-quadrado, considerando-se como estatisticamente significativos os valores de $\mathrm{p}$ menores que $5 \%$.

O projeto de pesquisa foi aprovado pelo Comitê de Ética em Pesquisa da Faculdade de Ciências Médicas/Unicamp (com registro no 1063/2011).

\section{Resultados}

No período de junho de 2006 a dezembro de 2010, foram realizados 762 atendimentos a 745 mulheres (sendo que 17 deles foram de mulheres revitimizadas no período). Trinta e quatro mulheres foram excluídas porque a agressão não configurou violência sexual e 24 por serem menores de 12 anos. Avaliou-se os dados de 687 mulheres vítimas de violência sexual atendidas no Hospital da Mulher Prof. Dr. José Aristodemo Pinotti da Unicamp. A média de idade foi de 23,7 anos (mínima: 12, máxima: 85 anos), mediana de 20 anos.

A maioria era ativa, sendo que $41.6 \%$ estavam empregadas e $39,4 \%$ eram estudantes. A maioria referiu ter religião $(84,9 \%)$ e ter prática religiosa $(74,7 \%)$, sendo que $52,6 \%$ eram católicas e $40,7 \%$ evangélicas (Tabela 1).

A Tabela 2 apresenta a caracterização da violência sexual sofrida pelas pacientes avaliadas. 
Tabela 1

Distribuição das mulheres vítimas de violência sexual segundo características sociodemográficas e antecedentes.

\begin{tabular}{|c|c|c|}
\hline Variável & $\mathbf{n}$ & $\%$ \\
\hline \multicolumn{3}{|c|}{ Faixa etária (anos) $(n=687)$} \\
\hline$\leq 19$ & 326 & 47,4 \\
\hline$>19$ & 361 & 52,6 \\
\hline \multicolumn{3}{|c|}{ Cor da pele $(n=687)$} \\
\hline Branca & 513 & 74,6 \\
\hline Não branca & 174 & 25,4 \\
\hline \multicolumn{3}{|c|}{ Estado civil $(n=672)$} \\
\hline Solteira & 512 & 76,1 \\
\hline Casada & 109 & 16,2 \\
\hline Separada & 43 & 6,4 \\
\hline \multicolumn{3}{|l|}{ Prole $(n=649)$} \\
\hline Sem filhos & 446 & 68,7 \\
\hline Com filhos & 203 & 31,2 \\
\hline \multicolumn{3}{|c|}{ Escolaridade (anos) $(n=664)$} \\
\hline Analfabetas & 17 & 2,5 \\
\hline$\leq 8$ & 282 & 42,4 \\
\hline $9-11$ & 271 & 40,8 \\
\hline$\geq 12$ & 83 & 12,5 \\
\hline \multicolumn{3}{|c|}{ Situação profissional $(n=665)$} \\
\hline Empregada & 277 & 41,6 \\
\hline Desempregada & 40 & 6,0 \\
\hline Em benefício & 9 & 1,3 \\
\hline Dona de casa & 57 & 8,5 \\
\hline Estudante & 262 & 39,4 \\
\hline Outros & 20 & 3,0 \\
\hline \multicolumn{3}{|c|}{ Relação sexual prévia à violência $(n=661)$} \\
\hline Sim & 490 & 74,1 \\
\hline Não & 171 & 25,9 \\
\hline \multicolumn{3}{|c|}{ Doença crônica $(n=505)$} \\
\hline Sim & 123 & 24,3 \\
\hline Não & 382 & 75,7 \\
\hline \multicolumn{3}{|c|}{ Antecedente pessoal de violência sexual $(n=449)$} \\
\hline Sim & 73 & 16,2 \\
\hline Não & 376 & 83,8 \\
\hline \multicolumn{3}{|c|}{ Antecedente familiar de violência sexual $(n=379)$} \\
\hline Sim & 32 & 8,4 \\
\hline Não & 347 & 91,6 \\
\hline
\end{tabular}

As características do atendimento de emergência dispensado às mulheres encontram-se na Tabela 3. Dois terços das mulheres chegaram ao serviço nas primeiras 24 horas após a violência sexual e o atendimento nas primeiras 72 horas foi realizado em $87,6 \%$ das mulheres, permitindo a prescrição de profilaxias antirretroviral, vacinação/imunoterapia para hepatite $\mathrm{B}$, antibióticos e anticoncepção de emergência para a maioria das vítimas.
Com o objetivo de avaliar se houve mudanças ao longo do período estudado no que tange à busca precoce de atendimento após a violência sexual e à taxa de realização de $\mathrm{BO}$, foi realizada uma análise agrupando o período em dois biênios (Tabela 4).

A taxa de não adesão ao seguimento ambulatorial foi de $24,5 \%$. As pacientes que não aderiram (compareceram apenas ao atendimento de urgência) apresentaram, quando comparadas 
Tabela 2

Distribuição das mulheres vítimas de violência sexual em relação às características da violência

\begin{tabular}{|c|c|c|}
\hline Variável & $\mathbf{n}$ & $\%$ \\
\hline \multicolumn{3}{|c|}{ Faixa horária de ocorrência da agressão $(n=687)$} \\
\hline $18: 01$ às $24: 00$ & 240 & 34,9 \\
\hline 00:01 às 07:00 & 282 & 41,0 \\
\hline 07:01 às $18: 00$ & 92 & 13,3 \\
\hline Imprecisa & 73 & 10,6 \\
\hline \multicolumn{3}{|c|}{ Local da abordagem $(n=682)$} \\
\hline Rua & 285 & 41,7 \\
\hline Residência & 143 & 20,9 \\
\hline Ponto de ônibus & 67 & 9,8 \\
\hline Escola & 15 & 2,2 \\
\hline Trabalho & 9 & 1,3 \\
\hline Outros & 129 & 18,9 \\
\hline \multicolumn{3}{|c|}{ Relação com o agressor $(n=667)$} \\
\hline Desconhecido & 462 & 69,2 \\
\hline Conhecido & 205 & 30,7 \\
\hline \multicolumn{3}{|c|}{ Número de agressores $(n=657)$} \\
\hline Único & 575 & 87,5 \\
\hline Múltiplos & 81 & 12,3 \\
\hline \multicolumn{3}{|c|}{ Abordagem com intimidação $(n=647)$} \\
\hline Sim & 599 & 92,5 \\
\hline Não & 48 & 7,5 \\
\hline \multicolumn{3}{|c|}{ Tipo de intimidação ( $n=643$ ) } \\
\hline Arma de fogo & 157 & 24,4 \\
\hline Arma branca & 102 & 15,8 \\
\hline Força física & 323 & 50,2 \\
\hline Ameaça verbal & 135 & 21,0 \\
\hline Outros & 81 & 12,3 \\
\hline \multicolumn{3}{|c|}{ Tipo de agressão sexual $(n=581)$} \\
\hline Coito vaginal & 516 & 88,8 \\
\hline Coito oral & 181 & 31,2 \\
\hline Coito anal & 134 & 23,0 \\
\hline Mais de um tipo & 212 & 36,4 \\
\hline \multicolumn{3}{|c|}{ Realização de boletim de ocorrência $(n=661)$} \\
\hline Sim & 407 & 61,5 \\
\hline Não & 254 & 38,5 \\
\hline
\end{tabular}

às que aderiram ao atendimento ambulatorial, menor escolaridade $(\mathrm{p}=0,0268)$, antecedente pessoal de violência sexual $(\mathrm{p}<0,0001)$, doenças crônicas $(\mathrm{p}<0,0001)$, mais agressões por conhecido $(\mathrm{p}=0,044)$ e por meio de ameaça verbal $(\mathrm{p}=$ 0,0239). Essas vítimas dividiram menos com outras pessoas sobre a violência $(p=0,0001)$ e quando o fizeram não se sentiram apoiadas $(\mathrm{p}=$ 0,0210 ). O estupro representou, mais frequentemente, a primeira relação sexual para as pacientes que aderiram ao acompanhamento ambulatorial $(p=0,0436)$.

\section{Discussão}

Embora a subnotificação de casos de violência sexual seja elevada $4,18,19$, a prevalência e as consequências individuais e coletivas são suficientemente graves para torná-la um problema de saúde pública mas que vem sendo continuamente negligenciado 14 . Considerando isso, este estudo apresentou o perfil sóciodemográfico, relacionado à violência sexual e ao primeiro atendimento de 691 mulheres vítimas de violência sexual atendidas em um serviço universitário. 
Tabela 3

Descrição do atendimento de emergência dispensado às mulheres após a violência sexual.

\begin{tabular}{|c|c|c|}
\hline Variável & $\mathbf{n}$ & $\%$ \\
\hline \multicolumn{3}{|c|}{ Tempo transcorrido entre o evento e a chegada ao serviço $(n=687)$} \\
\hline Até 24 horas & 430 & 65,3 \\
\hline Acima de 24 horas & 228 & 34,7 \\
\hline \multicolumn{3}{|c|}{ Classificação do atendimento $(n=687)$} \\
\hline Imediato (até 5 dias) & 602 & 87,6 \\
\hline Tardio (após 5 dias) & 85 & 12,3 \\
\hline \multicolumn{3}{|c|}{ Prescrição profilaxia com antirretrovirais $(n=679)$} \\
\hline Sim & 571 & 84,0 \\
\hline Não & 108 & 16,0 \\
\hline \multicolumn{3}{|c|}{ Prescrição de antibióticos para DST $(n=683)$} \\
\hline Sim & 591 & 86,5 \\
\hline Não & 92 & 13,5 \\
\hline \multicolumn{3}{|c|}{ Prescrição de vacina/imunoglobulina para hepatite $B(n=682)$} \\
\hline Sim & 566 & 82,9 \\
\hline Não & 116 & 17,1 \\
\hline \multicolumn{3}{|c|}{ Prescrição de anticoncepção de emergência $(n=683)$} \\
\hline Sim & 497 & 72,7 \\
\hline Não & 186 & 27,3 \\
\hline \multicolumn{3}{|c|}{ Contou a alguém sobre a violência $(n=588)$} \\
\hline Sim & 585 & 95,7 \\
\hline Não & 3 & 4,3 \\
\hline \multicolumn{3}{|c|}{ Pessoas a quem contou sobre a violência $(n=585)$} \\
\hline Família $(n=456)$ & 412 & 90,4 \\
\hline Mãe $(n=407)$ & 350 & 86,0 \\
\hline Pai $(n=237)$ & 174 & 73,4 \\
\hline Cônjuge $(n=267)$ & 207 & 77,5 \\
\hline \multicolumn{3}{|c|}{ Sentiu-se apoiada $(n=529)$} \\
\hline Sim & 456 & 86,2 \\
\hline Não & 57 & 10,8 \\
\hline Parcialmente & 16 & 3,0 \\
\hline
\end{tabular}

Tabela 4

Distribuição do número de mulheres atendidas, tempo transcorrido entre a violência e o atendimento e realização de BO segundo agrupamentos de períodos avaliados.

\begin{tabular}{|c|c|c|c|c|c|c|}
\hline \multirow[t]{2}{*}{ Variáveis } & \multicolumn{2}{|c|}{ 2006-2008 } & \multicolumn{2}{|c|}{ 2009-2010 } & \multirow[t]{2}{*}{ Total } & \multirow[t]{2}{*}{ Valor de $\mathrm{p}$} \\
\hline & $\mathbf{n}$ & $\%$ & $\mathrm{n}$ & $\%$ & & \\
\hline Número de atendimentos & 381 & 55,5 & 306 & 44,5 & 687 & NA \\
\hline Atendimentos em até 24 horas & 220 & 60,6 & 210 & 71,2 & 430 & 0,005 * \\
\hline Atendimentos em até 72 horas & 298 & 82,1 & 263 & 89,2 & 561 & 0,011 * \\
\hline Realizou BO & 236 & 65,2 & 171 & 57,2 & 407 & 0,035 * \\
\hline
\end{tabular}

BO: Boletim de Ocorrência; NA: não aplicável.

* $p<0,05$. 
Do ponto de visto sóciodemográfico, o perfil encontrado, composto por adolescentes e mulheres jovens, brancas, solteiras, sem filhos, com escolaridade acima da média nacional e regional, hígidas e ativas, com religião e prática religiosa, foi compatível com estudos nacionais e internacionais 20,21,22,23,24,25. Entre as pacientes avaliadas, $16 \%$ já haviam sofrido violência sexual em outro momento da vida, dado inferior ao encontrado na literatura internacional 26. É importante ressaltar que a violência sexual foi a primeira relação sexual para um quarto das vítimas, e se considerarmos apenas pacientes com até 19 anos, esta taxa sobe para 45,4\%. Em Curitiba, num estudo realizado com 117 mulheres, cerca de um terço das vítimas (independente da faixa etária) não havia tido relação sexual anterior à violência sexual, sendo que $28,7 \%$ delas tinham idades entre 10 e 19 anos 22 . Consideramos que esse dado representa um potencial agravante à sexualidade futura dessas pacientes 27 .

Os dados referentes ao evento apontam que o estupro ocorreu principalmente no final do dia e na madrugada, na rua, perpetrado via vaginal, por agressor único, desconhecido, com intimidação, particularmente por força física. Esses dados são semelhantes aos encontrados em estudos realizados em Teresina (Piauí) 23 e Londrina (Paraná) 25 , que também registraram maior frequência de agressores desconhecidos, respectivamente, $51 \%$ e $58,5 \%$ das agressões. Todavia, esses dados revelam uma realidade pouco relatada na literatura, que em sua maioria descreve estudos com agressor conhecido e com relação íntima com a vítima. Constata-se que embora a maioria dos estudos nacionais 28 e internacionais $2,4,5$ ocupe-se de agressões intrafamiliares, violência doméstica e por parceiro íntimo, vêm crescendo a produção científica nacional sobre a agressão predominantemente urbana, perpetrada por agressor desconhecido e abordando a violência sexual especificamente e não esta no contexto de outros tipos de violência 22,23,25.

Outro aspecto importante refere-se à subnotificação à polícia, fenômeno apontado frequentemente na literatura nacional e internacional, que considera as taxas oficiais apenas a ponta de um iceberg 17,18 . No presente trabalho, pouco mais da metade das vítimas realizaram BO. Alguns estudos apontam que apenas de 8 a 10\% das vítimas reportam os crimes à polícia, sendo ainda menor a taxa entre adolescentes de ensino médio (5\%) 18,29; outros apresentam ampla variação para notificação de crimes sexuais (3,6 a 43\%) 17 . No Brasil, com a notificação compulsória por profissionais da saúde, potencialmente há mais dados 12 , porém este estudo não permite discutir tal possibilidade. A baixa notifica- ção policial pode contribuir para uma distorção da realidade no que concerne à real magnitude do problema e à estruturação e implementação adequada de políticas públicas, tanto para prevenção quanto para assistência.

Com relação ao contato com o serviço de saúde, este se deu precocemente, com a maioria dos atendimentos sendo classificados como imediato e com a prescrição de profilaxias para DST (antirretrovirais, antibióticos e vacina/imunoglobulina para hepatite B) e anticoncepção de emergência. $O$ contato precoce com o serviço de saúde é essencial para promover a melhor qualidade de atendimento quanto ao momento de introdução de profilaxia para DST e anticoncepção de emergência. Diminuir a frequência de infecções por DST e gestações decorrentes de estupro é diminuir o sofrimento e a possibilidade de uma nova agressão, dado o impacto que seria a necessidade de uma interrupção de gestação. O risco para infecção por DST depende, entre outros fatores, dos tipos de exposição sexual (vaginal, anal ou oral), do tempo de exposição (única, múltipla ou crônica), do número de agressores, exposição a secreções sexuais ou sangue e início precoce da profilaxia ARV (que pode ser instituída até 72 horas após o contato) ${ }^{12}$. As vítimas que se apresentavam pela primeira vez ao serviço após 72 horas; que seguramente não haviam tido penetração vaginal ou contato vaginal com esperma; que estavam grávidas no momento da avaliação; que estavam na menopausa ou que haviam se submetido à laqueadura anteriormente não receberam anticoncepção de emergência.

Em relação à adesão ao seguimento ambulatorial, mulheres que não aderiram apresentavam, quando comparadas àquelas que compareceram ao ambulatório, menor escolaridade, atividade sexual anterior à violência, doenças crônicas e antecedente pessoal de violência sexual, foram mais agredidas por conhecidos, dividiram menos frequentemente com outras pessoas sua vivência sobre a violência sexual e não se sentiram apoiadas quando o fizeram. Houve uma melhora das taxas de adesão em comparação com estudo conduzido anteriormente no serviço ${ }^{30}$. A experiência de violência sexual em si é um determinante para a decisão de buscar ajuda ou não, além da resposta que a rede de apoio oferece para as vítimas que contam sobre sua vivência 31,32 . Ruback et al. 33 verificaram que $67 \%$ das mulheres agredidas por parceiro íntimo e que procuraram um centro de atendimento à crise já haviam dividido com familiares ou amigos sobre a agressão anteriormente. As respostas e reações da família, amigos e sistema de justiça podem ser incapazes de gerar apoio e até mesmo causar maiores se- 
quelas nas vítimas, levando-as a internalizar as respostas e percepções daqueles com quem dividiram sua vivência 34,35 . Lempert 36 sugere que a busca por auxílio de amigos e família exigiria da vítima uma definição e uma resposta na sua possível percepção sobre responsabilização pela agressão, verificando se a percepção daquele que oferece cuidado estaria de acordo com o que é informado.

É importante apontar o que consideramos a principal limitação do estudo, o fato de que os dados aqui apresentados foram coletados com base em anotações dos atendimentos dos prontuários realizadas por diferentes profissionais, e algumas informações não estavam disponíveis por preenchimento incompleto das fichas de atendimento. Essa é uma dificuldade recorrente em estudos deste tipo, o que não invalida os achados, mas indica cautela na interpretação dos dados.

Os resultados encontrados, que delineiam o perfil das vítimas e o padrão da agressão reforçam a gravidade da situação, e a descrição do atendimento aponta a importância da abordagem ágil e de qualidade para acolhimento e seguimento desta demanda.

Esperamos que os dados apresentados contribuam para a ampliação de políticas de atenção às mulheres vítimas de violência sexual e auxiliem na otimização do cuidado e minimização do sofrimento ocasionado por este evento.

\section{Conclusão}

O perfil das pacientes vítimas de violência sexual atendidas por este serviço universitário de referência caracterizou-se por uma maioria de mu- lheres jovens, brancas, solteiras, em atividade profissional ou estudantes, com religião e prática religiosa. Um quarto das vítimas não tinha atividade sexual anterior à violência. A violência sexual ocorreu principalmente à noite, a partir de abordagem feita na rua, por agressor desconhecido, único e com intimidação. A maioria das pacientes chegou ao atendimento em tempo hábil para que medidas profiláticas fossem instauradas, além disto, durante o período estudado houve significativo crescimento da taxa de procura precoce. Compartilharem com alguém sobre a violência sexual e sentirem-se apoiadas correlaciona-se com a adesão ao atendimento ambulatorial por essas mulheres. Pacientes que não compareceram ao seguimento ambulatorial apresentavam menor escolaridade, atividade sexual anterior à violência, doenças crônicas, antecedente pessoal de violência sexual, mais agressões por conhecidos, menor compartilhamento com outras pessoas de sua vivência sobre a violência sexual e não se sentiram apoiadas quando o fizeram. É necessário que as equipes e profissionais responsáveis pelo atendimento de emergência estejam atentos para o perfil das pacientes associado a não adesão ambulatorial, para que sejam implementadas estratégias para aumentar seu comparecimento e assim proporcionar atendimento ótimo e adequado. Embora não seja possível avaliar o impacto da violência sexual no sofrimento psíquico ou o desenvolvimento ou não de sintomas nessa subpopulação, é possível inferir, pelos dados apresentados, que muitas delas necessitariam de apoio em saúde mental e, sem utilizarem o acompanhamento oferecido, podem desenvolver sequelas de longa duração. 


\section{Resumen}

La violencia sexual es un problema global de salud pública y se han implementado acciones para estimular estudios en el tema, a fin de proponer intervenciones de prevención y atención adecuadas. Este trabajo tuvo por objetivo caracterizar la población de mujeres que sufrió violencia sexual, y describir las características de la agresión y de la atención dispensada en un servicio universitario de referencia. Se trata de un estudio cuantitativo y retrospectivo sobre la atención por violencia sexual de junio de 2006 a diciembre de 2010. Se evaluaron a 687 mujeres, la mayoría blanca, soltera, sin hijos, con una edad media de 23,7 años, escolaridad entre básica y media, con empleo, con religión y practicantes. Un cuarto sin relación sexual anterior. La violencia sexual principalmente se produce por la noche, en la calle, cometida por un agresor desconocido y único, vía vaginal y con intimidación. La mayoría se lo contó a otras personas y se sintió apoyada. Hubo atención precoz para casi un $90 \%$ de las mujeres, estableciendo medidas profilácticas. Se produjo un aumento de la búsqueda temprana del servicio a lo largo del período. Conocer mejor las características de la población y de los hechos puede auxiliar en la estructuración y cualificación de modelos de atención.

Violencia contra la Mujer; Violación; Violencia Sexual; Delitos Sexuales

\section{Referências}

1. Oliveira EM, Barbosa RM, Moura AAVM, Kossel K, Morelli K, Botelho LFF, et al. Atendimento às mulheres vítimas de violência sexual: um estudo qualitativo. Rev Saúde Pública 2005; 39:376-82.

2. Krug EG, Dahlberg LL, Mercy JA, Zwi AB, Lozano R, editors. World report on violence and health. Geneva: World Health Organization; 2002.

3. Ministério da Saúde. Temático prevenção de violências e cultura da paz III. Brasília: Organização Pan-Americana da Saúde; 2008

4. Black MC, Basile KC, Breiding MJ, Smith SG, Walters ML, Merrick MT, et al. The National Intimate Partner and Sexual Violence Survey (NISVS): 2010 summary report. Atlanta: National Center for Injury Prevention and Control, Centers for Disease Control and Prevention; 2011.

5. Basile KC, Smith SG. Sexual violence victimization of women: prevalence, characteristics, and the role of public health and prevention. Am J Lifestyle Med 2011; 5:407-17.

\section{Colaboradores}

C. O. Facuri participou da concepção e projeto, coleta de dados, análise e interpretação dos dados e resultados, redação do artigo, revisão crítica relevante do conteúdo intelectual, discussão dos dados e aprovação do conteúdo final a ser publicado. A. M. S. Fernandes participou da concepção e projeto, análise e interpretação dos dados, redação do artigo e aprovação final da versão a ser publicada. K. D. Oliveira e T. S. Andrade participaram da concepção e projeto, coleta dos dados, revisão crítica do conteúdo intelectual e aprovação final da versão a ser publicada. R. C. S. Azevedo participou da concepção e projeto, análise e interpretação dos dados, redação do artigo, revisão crítica relevante do conteúdo intelectual, aprovação final da versão a ser publicada.

\section{Agradecimentos}

Ao psiquiatra Amilton dos Santos Júnior, pelo auxílio na análise estatística dos dados.
6. Brasil. Lei no 12.015 , de 7 de agosto de 2009. Altera o Título VI da Parte Especial do Decreto-Lei no 2.848, de 7 de dezembro de 1940 - Código Penal, e o art. 1o da Lei no 8.072, de 25 de julho de 1990, que dispõe sobre os crimes hediondos, nos termos do inciso XLIII do art. 5o da Constituição Federal e revoga a Lei no 2.252, de 10 de julho de 1954, que trata de corrupção de menores. Diário Oficial da União 2009; 10 ago.

7. Mason F, Lodrick Z. Psychological consequences of sexual assault. Best Pract Res Clin Obstet Gynaecol 2013; 27:27-37.

8. Grossin C, Sibille I, Grandmaison GL, Banasr A, Brion F, Durigon M. Analysis of 418 cases of sexual assault. Forensic Sci Int 2003; 131:125-30.

9. Favarelli C, Giugni A, Salvatori S, Ricca V. Psychopathology after rape. Am J Psychiatry 2004; 161:1483-5. 
10. Campbell L, Keegan A, Cybulska B, Forster G. Prevalence of mental health problems and deliberate self-harm in complainants of sexual violence. J Forensic Leg Med 2007; 14:75-8.

11. United Nations General Assembly. Declaration on the elimination of violence against women. Geneva: United Nations; 1993.

12. Departamento de Ações Programáticas Estratégicas, Secretaria de Atenção à Saúde, Ministério da Saúde. Prevenção e tratamento dos agravos resultantes da violência contra mulheres e adolescentes: norma técnica. 3ạ Ed. Brasília: Ministério da Saúde; 2010.

13. DeGue S, Simon TR, Basile KC, Yee SL, Lang K, Spivak $\mathrm{H}$. Moving forward by looking back: reflecting on a decade of CDC's work on sexual violence prevention, 2000-2010. J Womens Health (Larchmt) 2012; 21:1211-8.

14. Bedone AJ, Faúndes A. Atendimento integral às mulheres vítimas de violência sexual: Centro de Assistência Integral à Saúde da Mulher, Universidade Estadual de Campinas. Cad Saúde Pública 2007; 23:465-9.

15. Higa R, Mondaca ADCA, Reis MJ, Lopes MHBM. Atendimento à mulher vítima de violência sexual: Protocolo de Assistência de Enfermagem. Rev Esc Enferm USP 2008; 42:377-82.

16. Maia CAT, Mondaca ADCA, Duarte CS, Lima JA Colodo M, Lemos TM, et al. Mulheres vítimas de violência sexual: atendimento multidisciplinar Femina 2000; 28:155-61.

17. Coordenadoria de Análise e Planejamento, Secretaria da Segurança Pública de São Paulo. Estatística de criminalidade. Manual de interpretação. http://www.ssp.sp.gov.br/estatistica/downloads/ manual.pdf (acessado em 11/Ago/2010).

18. Rand M, Catalano S. Criminal victimization, 2006. http://www.ojp.usdoj.gov/bjs/pubalp2.htm\#cvus (acessado em 06/Jun/2010).

19. Madi SRC, Knob LF, Lorencetti J, Marcon NO, Madi JM. Sexual violence: experience of a Program for the Care of Sexual Violence Victims (PRAVIVIS) at a General Hospital in Caxias do Sul, South Brazil. Rev AMRIGS 2010; 54:13-8.

20. Danielson CK, Holmes MM. Adolescent sexual assault: an update of the literature. Curr Opin Obste Gynecol 2004; 16:383-8.

21. Mattar R, Abraão AR, Andalaft Neto J, Colas OR, Schroeder I, Machado SJR, et al. Assistência multiprofissional à vitima de violência sexual: a experiência da Universidade Federal de São Paulo. Cad Saúde Pública 2007; 23:459-64.

22. Andrade RP, Guimarães ACP, Fagoti Filho A, Carvalho NS, Arrabal JS, Rocha DM, et al. Características demográficas e intervalo para atendimento em mulheres vítimas de violência sexual. Rev Bras Ginecol Obstet 2001; 23:583-7.

23. Lopes IMRS, Gomes KRO, Silva BB, Deus MCBR, Galvão ERCGN, Borba DC. Caracterização da violência sexual em mulheres atendidas no projeto Maria-Maria em Teresina-PI. Rev Bras Ginecol Obstet 2004; 26:111-6.
24. Instituto Brasileiro de Geografia e Estatística. Re sultados gerais da amostra do Censo Demográfico de 2010. http:/ / www.censo2010.ibge.gov.br/amos tra/ (acessado em 25/Jul/2012).

25. Oliveira PM, Carvalho MLO. Perfil das mulheres atendidas no Programa Municipal de Atendimento à Mulher Vítima de Violência Sexual em LondrinaPR e as circunstâncias da violência sofrida: perío do de outubro de 2001 a agosto de 2004. Semina Ciênc Biol Saúde 2006; 27:3-11.

26. Coid J, Petruckevitch A, Chung WS, Richardson J, Moorey S, Feder G. Abusive experiences and psychiatric morbidity in women primary care attenders. Br J Psychiatry 2003; 183:332-9.

27. Filkenhor D, Ormrod R, Turner H, Hamby SL. The victimization of children and youth: a comprehen sive, national survey. Child Maltreat 2005; 10:5-25.

28. Guimarães JATL, Villela WV. Características da violência física e sexual contra crianças e adolescentes atendidos no IML de Maceió, Alagoas, Brasil. Cad Saúde Pública 2011; 27:1647-53.

29. Belknap J. Rape: too hard to report and too easy to discredit victims. Violence Against Women 2010; 16:1335-44.

30. Oshikata CT, Bedone AJ, Papa MSF, Santos GB, Pinheiro CD, Kalies AH. Características das mulheres violentadas sexualmente e da adesão ao seguimento ambulatorial: tendências observadas ao longo dos anos em um serviço de referência em Campinas, São Paulo, Brasil. Cad Saúde Pública 2011; 27:701-13.

31. Kaukinen CE, DeMaris A. Age at first sexual assault and current substance use and depression. J Interpers Violence 2005; 20:1244-70.

32. Ahrens CE, Campbell R. Assisting rape victims as they recover from rape: the impact of friends. J Interpers Violence 2000; 15:959-86.

33. Ruback RB, Ivie DL. Prior relationship, resistance and injury rapes: an analysis of crisis center records. Violence Vict 1988; 3:99-111.

34. Wortman C, Lehman D. Reactions to victims of life crisis: support attempts that fail. In: Sarason I Sarason B, editors. Social support: theory, research, and applications. Boston: Martinus Nijhoff; 1985. p. 463-89.

35. Taylor S, Wood J, Lichtman RR. It could be worse: selective evaluation as a response to victimization. J Soc Issues 1983; 39:19-40.

36. Lempert L. Women's strategies for survival: developing agency in abusive relationships. J Fam Violence 1996; 11:269-89.

Recebido em 06/Set/2012

Versão final reapresentada em 24/Dez/2012

Aprovado em 11/Jan/2013 